\title{
Enfoque diagnóstico del paciente con rubor facial transitorio en atención primaria
}

\section{A Diagnostic Approach of Patient with Flushing in Primary Care}

\author{
José Manuel Vivas Prieto \\ Pontificia Universidad Javeriana, Colombia \\ Sandra Virginia Caballero Vera ${ }^{\mathrm{b}}$ \\ Pontificia Universidad Javeriana, Colombia \\ Mateo Echeverry Ramírez ${ }^{c}$ \\ Pontificia Universidad Javeriana, Colombia
}

a Coordinador académico de programa de Medicina Familiar, Pontificia Universidad Javeriana (PUJ), Bogotá, Colombia. Profesor del Departamento de Medicina Preventiva y Social, Facultad de Medicina (PUJ).

b Residente de Medicina Familiar, Facultad de Medicina, Pontificia Universidad Javeriana, Bogotá, Colombia.

c Residente de Medicina Familiar, Facultad de Medicina, Pontificia Universidad Javeriana, Bogotá, Colombia.

\section{RESUMEN}

El rubor facial transitorio se define como una sensación de calor, acompañada de enrojecimiento de la piel, causada por una respuesta vasomotora con el resultante incremento en el flujo sanguíneo regional, debido a la acción directa de diferentes mediadores liberados por los nervios. Los cuadros pueden ser episódicos o constantes y se relacionan con respuestas fisiológicas y con patologías de origen benigno y maligno. El objetivo de la revisión es realizar un abordaje racional de esta condición en el escenario de atención primaria, con el fin de llegar a un diagnóstico precoz y completo, abordando los principales diagnósticos diferenciales asociados con esta condición.

Palabras clave

rubor; sofocos; manifestaciones clínicas; diagnóstico diferencial; prevención primaria.

\begin{abstract}
The definition of flushing involves a sensation of heat along with redness of the skin, caused by vasomotor influx resulting in an increased of the regional vascular blood flow due to the direct action of different mediators through the vasomotor nerves. These symptoms can be episodic or repetitive and they can be related to benign or malignant conditions. The objective of this review is to develope a rational approach of the condition at primary care, with the purpose of an early and complete diagnosis, covering the main differential diagnosis related to this condition.

Keywords

flushing; blushing; hot flashes; signs and symptoms; diagnosis; differential; primary prevention.
\end{abstract}

\section{Introducción}

Los episodios de rubor facial transitorio representan un motivo de consulta frecuente para los médicos de atención primaria. Ocasionalmente, es un síntoma lo suficientemente mórbido como para afectar la calidad de vida de quienes lo padecen. Sin embargo, no hay unanimidad en el abordaje
Cómo citar: Vivas Prieto JM, Caballero Vera SV, Echeverry Ramírez M. Enfoque diagnóstico del paciente con rubor facial transitorio en atención primaria. Univ. Med. 2019;60(1). doi: https://doi.org/10.11144/Javeriana.umed60-1.edpr 
tanto diagnóstico como terapéutico, a lo que se suma la frecuente trivialización de los síntomas por parte del propio afectado y del médico. Los episodios de rubor facial secundarios a condiciones benignas como rosácea y menopausia han demostrado influir negativamente en la autoestima, en la calidad de vida, en la productividad laboral, en las relaciones interpersonales y en costos económicos para el paciente $(1,2,3)$. La aparición de rubor facial -usualmente confundido con trastornos funcionales- puede ser una clave frecuentemente pasada por alto para el diagnóstico temprano de algunas neoplasias neuroendocrinas (4). En un estudio retrospectivo en población estadounidense, en 663 pacientes encuestadas con síndrome carcinoide, se encontró que consultaron frecuentemente por episodios de diarrea y rubor facial, y que estos síntomas afectaban su calidad de vida, desempeño laboral y socialización (2).

Las diferentes opciones diagnósticas que existen sustentan la importancia de un adecuado abordaje del rubor facial en atención primaria que permita el manejo oportuno de las enfermedades que lo producen e influir positivamente en los desenlaces de cada una y en la calidad de vida de los pacientes.

\section{Metodología}

Se hizo una búsqueda de la literatura en bases de datos usando los términos flushing, blushing, rubor facial, incluyendo guías de práctica clínica, revisiones sistemáticas, metanálisis, revisión de temas y estudios observacionales. Las bases seleccionadas fueron Pubmed, Embase y SciELO. Se consideraron publicaciones desde 2000 hasta 2017, y se seleccionaron artículos relevantes en cuanto a presentación clínica, diagnóstico y tratamiento, para llevar a cabo una revisión narrativa del abordaje en atención primaria del rubor facial transitorio.

\section{Presentación clínica}

El rubor facial transitorio (flushing, en inglés) se refiere a la ocurrencia de episodios caracterizados por sensación de calor, acompañados de enrojecimiento visible de la piel, que afectan predominantemente la región facial, el cuello y, en menor medida, la región anterior del tronco, el epigastrio y las extremidades superiores (2). El término también abarca esta misma sintomatología cuando es de origen emocional o psíquico y sería equivalente al sonrojo (blushing).

El rubor facial transitorio puede ser episódico o persistente. El primero consiste en la aparición ocasional de los síntomas, con largos periodos sin afectación de la piel; en el segundo, la presencia de episodios repetitivos puede llevar a una afectación de la piel entre los ataques, presentándose eritema facial persistente asociado a telangiectasias. En los ataques episódicos puede haber un desencadenante de tipo farmacológico, alimentario, emocional o ambiental, con una duración de segundos a minutos; mientras que los ataques repetitivos pueden durar desde minutos a horas $y$, usualmente, son desencadenados por patologías cutáneas o sistémicas. El rubor facial transitorio puede clasificar como húmedo y seco, este último se subclasifica según la presencia o no de disestesias (tabla 1) (4). Las manifestaciones sistémicas (hipo/hipertensión, síntomas digestivos y palpación de masas abdominales) desempeñan un papel clave en el diagnóstico diferencial, así como en la concomitancia de otros signos dermatológicos (5).

Tabla 1

Clasificación del rubor facial

Mediado por el SNC (rubor húmedo o wet flushing) Mediado por vasodilatación simple (rubor seco o dry flushing)

Mediado por un mecanismo sensorial antidrómico (disestesia)

Secundario a un vasodilatador circulante (no disestesia)

Fuente: adaptado de Batalla Cebey A, De la

TF. Signos guía/diagnóstico diferencial: Rubor facial transitorio. Metodología diagnóstica y tratamiento. Piel. 2012; 27:390-400. 


\section{Prevención}

No es posible determinar estrategias de prevención primaria al tratarse de una condición con un amplio abanico de causas, cuya característica en común es la presencia de rubor facial transitorio; por esta razón, se recalca la importancia de realizar un abordaje diagnóstico cuidadoso. De forma general, se puede disminuir la frecuencia y la severidad de los episodios de rubor facial transitorio, recomendando modificaciones en los estilos de vida relacionados con factores emocionales, consumo de ciertos alimentos o exposiciones ambientales que desencadenen la sintomatología (5). Por último, cuando un paciente consulta por rubor facial transitorio, existe la oportunidad de realizar actividades de prevención primaria y secundaria para disminuir la carga de enfermedad de condiciones cardiovasculares y metabólicas asociadas concomitantemente con este problema (6).

\section{Diagnóstico}

El diagnóstico diferencial del rubor facial transitorio es extenso, y engloba tanto procesos benignos como malignos. Ello implica que no es posible realizar un algoritmo diagnóstico que aplique a todos los pacientes; tampoco es recomendable una batería inicial de pruebas diagnósticas para todos ellos. Por tanto, la clave es realizar una historia clínica completa con la cual tener una sospecha fundamentada para solicitar las pruebas diagnósticas más pertinentes de manera individualizada $(5,7)$. Se sugiere tener en cuenta los siguientes aspectos:

1. Factores desencadenantes: para poder identificar estos factores es útil que el paciente realice un diario de las dos últimas semanas, en especial se debe hacer hincapié en alimentos, bebidas alcohólicas y fármacos. $\mathrm{Si}$ bien los mencionados pueden, por sí mismos, generar episodios de rubor facial, también pueden bajar el umbral de respuesta a sustancias vasoactivas endógenas (5).

2. Características de las lesiones cutáneas: es importante distinguir el tiempo de aparición y desaparición del rubor facial, si se acompaña de sudoración o no (húmedo o seco), si son lesiones confluentes o en parches, el color de la piel durante el episodio de rubor facial (rojo intenso o marrón, que son característicos del síndrome carcinoide), si este se asocia a cianosis o palidez (5).

3. Características asociadas: se debe indagar por síntomas asociados durante los episodios de rubor: respiratorios, digestivos, hipertensión o hipotensión, cefalea, edema facial, urticaria o síntomas oculares, como ojo rojo o sensación de cuerpo extraño (5).

Basado en lo anterior, es importante para el médico de atención primaria mantener un alto grado de sospecha de enfermedades sistémicas ante episodios de rubor facial transitorio, especialmente cuando son prolongados, extensos, acompañados de habones persistentes, afectación extracutánea y concomitancia con palpación de masas abdominales.

Para el abordaje diagnóstico es útil considerar el rubor facial transitorio húmedo (mediado neurológicamente) que se manifiesta clínicamente asociado a sudoración, cuyas causas pueden ser en la mayoría de los casos fisiológicas, asociadas a emociones o por hipogonadismo (especialmente menopausia). El rubor facial transitorio seco se asocia con liberación de sustancias vasoactivas $y$, por tanto, a veces se acompaña de síntomas cardiovasculares. Las causas más frecuentes son los tumores, especialmente neuroendocrinos, mastocitosis e ingesta de algunos medicamentos.

Por otro lado, los principales diagnósticos diferenciales se pueden clasificar en no oncológicos y oncológicos (tablas 2 y 3 ). 
Tabla 2

Diagnósticos diferenciales no oncológicos

\begin{tabular}{|c|c|c|c|}
\hline \begin{tabular}{|l|} 
Diagnóstico \\
diferencial
\end{tabular} & \begin{tabular}{|l|l|}
$\begin{array}{l}\text { Puntos clave en la } \\
\text { historia clinica }\end{array}$ \\
\end{tabular} & $\begin{array}{l}\text { Puntos clave en el } \\
\text { examen físico }\end{array}$ & \begin{tabular}{|l|} 
Exámenes \\
laboratorio
\end{tabular} \\
\hline Rosácea & & $\begin{array}{l}\text { Pápulas, pústulas y } \\
\text { telangiectasias } \\
\text { limitadas a la } \\
\text { región facial, } \\
\text { eritema facial } \\
\text { persistente } \\
\text { Rubor facial seco }\end{array}$ & Ninguno \\
\hline Menopausia & $\begin{array}{l}\text { Aparición en mujeres } \\
5^{\mathrm{a}} \text { a } 7^{\mathrm{a}} \text { década de la } \\
\text { vida }\end{array}$ & Ninguno específico & Ninguno \\
\hline $\begin{array}{l}\text { Reacciones alérgicas } \\
\text { tipo } \\
\text { urticaria/angioedema } \\
\text { agudo }\end{array}$ & & $\begin{array}{lr}\text { Presencia } & \text { de } \\
\text { habones (duración } \\
\text { menor a } 72 \text { horas), } \\
\text { que r suelen } \\
\text { desaparecer y } \\
\text { reaparecer incluso } \\
\text { hasta por } 6 \text { meses } \\
\text { Rubor facial seco }\end{array}$ & Según severidad \\
\hline $\begin{array}{l}\text { Reacción tipo rubor } \\
\text { facial asociada con } \\
\text { procesos fisiológicos }\end{array}$ & \begin{tabular}{|ll}
$\begin{array}{l}\text { Ejercicio, } \\
\text { picantes, }\end{array}$ & comida \\
emociones & alcohol, \\
\end{tabular} & 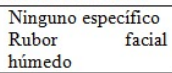 & Ninguno \\
\hline \begin{tabular}{|l|} 
Neurológico \\
\end{tabular} & \begin{tabular}{|l|} 
Relacionado con: \\
Migraña \\
Síndrome de Frey \\
(arlequín) \\
Síndrome de Homer \\
Hiperreflexia \\
autonómica
\end{tabular} & 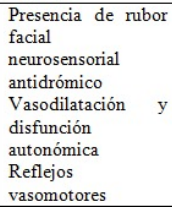 & $\begin{array}{l}\text { Dependiendo de la } \\
\text { causa }\end{array}$ \\
\hline
\end{tabular}

Tabla 3

Diagnósticos diferenciales oncológicos

\begin{tabular}{|c|c|c|c|}
\hline \begin{tabular}{|l|} 
Diagnósticos \\
diferenciales
\end{tabular} & $\begin{array}{l}\text { Puntos clave en la } \\
\text { historia clinica }\end{array}$ & \begin{tabular}{|l|}
$\begin{array}{l}\text { Puntos clave en el examen } \\
\text { fisico }\end{array}$ \\
\end{tabular} & \begin{tabular}{|l|} 
Exámenes laboratorio \\
\end{tabular} \\
\hline $\begin{array}{l}\text { Síndrome } \\
\text { carcinoide }\end{array}$ & $\begin{array}{l}\text { Diarrea } \\
\text { Broncoconstricción }\end{array}$ & $\begin{array}{l}\text { Rubor facial marrón rojizo o } \\
\text { rojo brillante } \\
\text { Rubor más allá de sitios } \\
\text { anatómicos usuales } \\
\text { Rubor facial seco }\end{array}$ & $\begin{array}{l}\text { S 5-HIAA urinario en } \\
24 \text { horas }\end{array}$ \\
\hline \begin{tabular}{|l|} 
Feocromocitoma \\
\end{tabular} & & \begin{tabular}{|l|} 
Hipertensión (sostenida \\
episódica) \\
Rubor facial seco
\end{tabular} & $\begin{array}{l}\text { Metanefrinas en orina } \\
24 \text { horas }\end{array}$ \\
\hline $\begin{array}{l}\text { Cáncer medular } \\
\text { de tiroides }\end{array}$ & $\begin{array}{l}\text { Historia personal o } \\
\text { familiar de } \\
\text { neoplasias } \\
\text { endocrinas } \\
\text { múltiples }\end{array}$ & $\begin{array}{l}\text { Rubor facial prolongado } \\
\text { Telangiectasias en cara y } \\
\text { brazos } \\
\text { Rubor facial seco }\end{array}$ & $\begin{array}{l}\text { Ecografía tiroides } \\
\text { Biopsia con punción } \\
\text { por aspiración con } \\
\text { aguja fina }\end{array}$ \\
\hline \begin{tabular}{|l|} 
Vipoma \\
pancreático
\end{tabular} & & Ninguno en especifico & Niveles VIP séricos \\
\hline $\begin{array}{lr}\begin{array}{l}\text { Carcinoma } \\
\text { células }\end{array} \\
\text { renales }\end{array}$ & & Masa abdominal & $\begin{array}{llr}\begin{array}{l}\text { Ecografía } \\
\text { urinarias }\end{array} & \text { de } & \text { vías } \\
\text { TAC } & \text { abdomen } \\
\text { contrastado } & \text { estudio } \\
\text { elección } & & \\
\end{array}$ \\
\hline Mastocitosis & & $\begin{array}{l}\text { Signo de Darier } \\
\text { Hipotensión } \\
\text { Rubor facial seco }\end{array}$ & $\begin{array}{l}\text { Biopsia, resiones } \\
\text { cutáneas o médula ósea } \\
\text { según afectación, frotis } \\
\text { de sangre periférica, } \\
\text { triptasas sérica, } \\
\text { citometria de flujo }\end{array}$ \\
\hline $\begin{array}{|lr|}\text { Secundario } & \text { a } \\
\text { tratamiento } & \\
\text { hormonal } & \text { en } \\
\text { pacientes r r con } \\
\text { cáncer de próstata } \\
\end{array}$ & & $\begin{array}{l}\text { Diaforesis posterior al } \\
\text { episodio } \\
\text { Rubor facial seco }\end{array}$ & Ninguno \\
\hline
\end{tabular}

\section{Etiología no oncológica}

\section{Rubor facial transitorio relacionado con emociones}

El rubor facial emocional (blushing) puede ser una respuesta fisiológica, especialmente en personas de piel clara (8). A veces puede aparecer sin que sea evidente un estímulo desencadenante. Los pacientes que presentan rubor facial emocional experimentan episodios frecuentes e intensos de enrojecimiento facial súbito e incontrolable provocado por estímulos emocionales o sociales (8). Lo más característico de este síndrome es que aparece de forma rápida y, con frecuencia, se acompaña de sensación de inquietud, calor en la cara, hormigueo y, en ocasiones, bloqueo mental (8). Habitualmente, abarca las mejillas y las orejas, pero puede extenderse hasta el cuello y el área anterior del tórax (8). A medida que se repite en diversas situaciones, puede afectar la calidad de vida y relaciones interpersonales de la persona (8).

El tratamiento inicial del rubor facial emocional consiste en terapia cognitivoconductual y entrenamiento en concentración de tareas (9), junto con un maquillaje adecuado y la utilización de ropa que cubra el cuello (10). Cuando esto es insuficiente, se puede intentar un abordaje farmacológico que incluye betabloqueadores, ansiolíticos, inhibidores selectivos de la recaptación de serotonina y otros fármacos antidepresivos (10). Eventualmente, los betabloqueadores pueden reducir el rubor facial emocional. Si bien un estudio determinó que lo hacían en aproximadamente un $75 \%$ de los pacientes, aún faltan más datos sobre su eficacia (11). Los ansiolíticos y los antidepresivos pueden ayudar a mejorar la ansiedad que produce el enrojecimiento; pero no hay estudios que demuestren su efectividad en la intensidad del rubor facial emocional (10).

Hasta el momento, solo se han publicado dos estudios sobre la toxina botulínica tipo A en personas con este problema, que mostraron la existencia de un alivio temporal; sin embargo, es una opción terapéutica limitada por el alto costo a largo plazo (10). La simpatectomía endoscópica transtorácica se ha usado en el rubor facial emocional incapacitante o en casos refractarios, con tasas de éxito de entre el $80 \%$ y el $90 \%$, aunque pueden darse complicaciones hasta en el $10 \%$ de los casos $(8,10)$. No hay una indicación clara para optar por el manejo quirúrgico, por lo que la evaluación de la severidad por parte del paciente es la única justificación relativa (8). 


\section{Rosácea}

Es una enfermedad inflamatoria crónica y frecuentemente progresiva. Se caracteriza por presentar una o varias de las siguientes lesiones en la región centrofacial: eritema persistente, telangiectasias, pápulas o pústulas e hiperplasia sebácea $(7,12)$, aunque también puede afectar áreas como cuero cabelludo, cuello, pabellón auricular, tórax anterior u ojos (13); además, se puede presentar con ardor o prurito, sequedad en la piel y edema (13). La rosácea es más prevalente en personas mayores de 30 años y en mujeres $(13,14)$. El diagnóstico de los 4 tipos de rosácea (eritematotelangiectásica, papulopustulosa, fimatosa y ocular) es eminentemente clínico $(13,15)$. No se requieren pruebas de laboratorio para definir el diagnóstico (15).

Para la rosácea existen diferentes tipos de tratamientos tópicos, sistémicos y hasta intervenciones quirúrgicas (16). Dentro del tratamiento no farmacológico, se recomienda evitar factores desencadenantes como la exposición solar, el consumo de alimentos picantes, alcohol, bebidas calientes y estrés (17). Además, es importante el uso de fotoprotección permanente (13) y la aplicación de hidratantes.

\section{Menopausia}

Los denominados "sofocos" son comunes en mujeres que entran en esta etapa, y se han descrito hasta en un $85 \%$ de mujeres menopaúsicas e incluso en $55 \%$ de mujeres antes del inicio de la irregularidad menstrual (3). Los síntomas referidos secundarios al proceso menopaúsico incluyen sensaciones espontáneas de calor, generalmente en la región torácica, cuello y cara, a menudo asociados con eritema, transpiración y posterior enfriamiento. No es raro que se asocie con sintomatología de origen ansioso como palpitaciones, de duración entre 5 y 30 minutos. Entre los desencadenantes se encuentran ambientes cálidos, estrés, alimentos o bebidas calientes (18).
Esta misma sintomatología puede aparecer en la noche e interferir con el sueño.

La sintomatología descrita en el contexto de una mujer entre 40 y 60 años, sin sospecha de otra causa, sugiere el diagnóstico de manifestaciones vasomotoras menopaúsicas y no se requieren exámenes de laboratorio.

El curso y la duración de los sofocos son importantes para individualizar el tratamiento de los síntomas en la menopausia. La terapia hormonal es considerada apropiada para el manejo de los sofocos; pero es preciso determinar cuidadosamente la duración del tratamiento y la consistencia con las necesidades y riesgos. Además, los sofocos suelen reaparecer después del cese de la terapia hormonal: se ha informado hasta en un $87 \%$ el regreso de los síntomas, independientemente de la duración del uso hasta 5 años (19). Para sofocos leves, se sugiere mantener estilos de vida saludable, un ambiente fresco, realizar ejercicio regularmente, evitar consumo de alcohol y café (20). En algunos ensayos clínicos, la gabapentina $(21,22)$, la oxibutinina y la venlafaxina mostraron un efecto clínicamente significativo (20).

Existen hallazgos no conclusivos sobre la asociación entre la presencia de síntomas vasomotores y el desarrollo de enfermedad aterosclerótica, hipertensión arterial y diabetes mellitus tipo 2. Se ha postulado como sustrato fisiopatológico el hipoestrogenismo posmenopáusico como factor desencadenante de un estado de hiperactividad simpática, por mecanismos no esclarecidos (23). De ahí que existan argumentos preliminares que invitan en atención primaria a indagar de forma más acuciosa la presencia y severidad de los síntomas vasomotores en mujeres tanto en la transición hacia la menopausia como en las mujeres posmenopáusicas como un marcador temprano de riesgo cardiometabólico (24).

\section{Anafilaxia}

La anafilaxia es una reacción de hipersensibilidad generalizada que amenaza la vida, en la que ocurre una liberación de mediadores de basófilos 
José Manuel Vivas Prieto, Sandra Virginia Caballero Vera, Mateo Echeverry Ramírez.

y mastocitos con síntomas y signos a diferentes niveles, entre los cuales se incluye el rubor facial transitorio que es asociado a edema de las conjuntivas y la mucosa oral. El diagnóstico de anafilaxia es muy probable cuando se cumple cualquiera de los tres criterios (tabla 4) (25).

\section{Tabla 4}

\section{Criterios diagnósticos para anafilaxis}

\begin{tabular}{l} 
1. Instauración aguda (minutos a horas) con afectación cutánea de mucosas, o ambos, y \\
al menos uno de los siguientes: \\
Compromiso respiratorio (p. ej., disnea, sibilancia-broncoespasmo, estridor, disminución \\
del flujo espiratorio máximo, hipoxemia). \\
Presión arterial reducida o síntomas asociados de disfunción orgánica (p. ej., hipotonía, \\
síncope, incontinencia). \\
\hline 2. Dos o más de los siguientes que ocurren rápidamente después de la exposición a un \\
probable alérgeno: \\
Compromiso mucocutáneo (p. ej., urticaria generalizada, picazón, edema labios-lengua- \\
úvula). \\
Compromiso respiratorio (p. ej., disnea, sibilancia-broncoespasmo, estridor, disminución \\
del flujo espiratorio máximo, hipoxemia). \\
Presión arterial reducida o síntomas asociados de disfunción orgánica (p. ej., hipotonía, \\
síncope, incontinencia). \\
Síntomas gastrointestinales persistentes (p. ej., dolor abdominal tipo cólico, vómitos). \\
\hline 3. Reducción de la presión arterial después de la exposición al alérgeno conocido para el \\
paciente: \\
Infantes y niños: presión arterial sistólica baja (según parámetros específicos para la \\
edad), o una disminución en la presión arterial sistólica mayor del $30 \%$. \\
Adultos: presión arterial sistólica inferior a 90 mmHg o una disminución superior al \\
$30 \%$ de la línea de base del paciente.
\end{tabular}

Fuente: adaptado de Hernández L, Papalia S, Pujalte GGA. Anaphylaxis. Prim Care Clin Off Pract. 2016;43(3):477-85.

Las pruebas de laboratorio respaldan el diagnóstico clínico; pero resultados normales no lo descartan (25). Las principales pruebas indicadas en la literatura son las concentraciones de triptasa sérica e histamina sérica elevadas, tomadas de forma inmediata en el servicio de urgencias, por lo que su disponibilidad se limita al contexto hospitalario (25).

\section{Rubor facial transitorio inducido por alcohol}

Es secundario a un metabolismo ausente o deficiente de acetaldehído deshidrogenasa, un metabolito hepático del alcohol etílico. La acumulación de acetaldehído da como resultado la degranulación de los mastocitos, liberación de histamina y síntomas de rubor facial, taquicardia y náuseas después de la ingesta de alcohol (26). Es frecuente en raza asiática y raro en otros grupos raciales (8). Puede ser potenciado por el uso concomitante de fármacos como griseofulvina, metronidazol, tacrolimus tópico, clorpropamida, o exacerbar la aparición de rubor facial en condiciones como rosácea, tumores carcinoides, mastocitosis, linfoma tipo Hodking y estados hipereosinofílicos (8). Eminentemente, el diagnóstico es clínico, y se confirma, en contextos de estudios de investigación, con el uso de parches de alcohol deshidrogenasa tras la ingesta de alcohol con la subsecuente mejoría de los episodios de rubor facial (8). En la tabla 5 se listan los medicamentos relacionados con rubor facial.

\section{Tabla 5}

Medicamentos relacionados con rubor facial

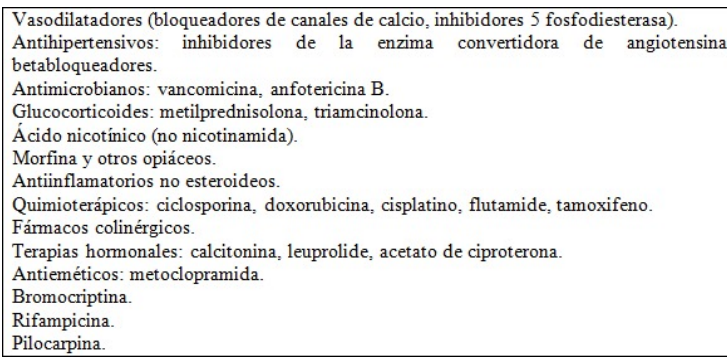

Quimioterápicos: ciclosporina, doxorubicina, cisplatino, flutamide, tamoxifeno

Eármacos colinérgicos.

Terapias hormonales: calcitonina, leuprolide, acetato de ciproterona.

Antieméticos: metoclopramida.

Bromocriptina.

Rifampicina.

Pilocarpina.

Fuente: adaptado de Batalla Cebey A, De la TF. Signos guía/diagnóstico diferencial: Rubor facial transitorio. Metodología diagnóstica y tratamiento. Piel. 2012;27:390-400.

\section{Etiología oncológica}

\section{Sindrome carcinoide}

Muchos tumores neuroendocrinos son asintomáticos y su hallazgo puede ser incidental. Se originan en el intestino delgado, en el recto, en el pulmón, etc., y sus características clínicas dependen de sus productos de secreción, como la serotonina y la sustancia P (27). En el síndrome carcinoide, una de las manifestaciones más comunes es el rubor facial; cuando el tumor se localiza en los bronquios, el rubor facial se caracteriza por una coloración rosarojizo brillante, confluente, que afecta la mayor parte del cuerpo y puede persistir durante horas o días (28); en el carcinoide gástrico, tiene apariencia rojiza-marrón, es pruriginoso y se disemina por todo el cuerpo. También se puede presentar diarrea (20\%), hepatomegalia (15\%), sudoración, hemoptisis, sibilancias e hipotensión (27).

El síndrome carcinoide se diagnóstica al medir en una orina de 24 horas las concentraciones 
ácido 5-hidroxiindolacético (5-HIAA), un metabolito importante de la serotonina $(5-\mathrm{HT})$ : valores de 5-HIAA dos veces por encima de lo normal son muy sospechosos de síndrome carcinoide $(4,7)$. En la presencia de un síndrome carcinoide, la sensibilidad y especificidad del 5-HIAA es del orden del $70 \%$ y del $90 \%$, respectivamente (28).

La tomografía computarizada, la resonancia magnética y la angiografía selectiva de la región abdomino-pélvica se deberían realizar para identificar y localizar el tumor y sus metástasis; pero en algunos casos el tumor primario solo puede ser identificado por medio de laparotomía (4).

\section{Feocromocitoma}

El feocromocitoma es un tumor productor de catecolaminas. Los principales signos y síntomas incluyen hipertensión, palpitaciones, cefalea, sudoración y palidez (20). De manera menos frecuente puede presentarse fatiga, náuseas, pérdida de peso, estreñimiento, rubor facial y fiebre (20). El rubor facial en pacientes con feocromocitoma generalmente dura de 10 a 45 minutos, y sugiere que el tumor es productor de epinefrina, dopamina o un mediador concomitante, como el péptido intestinal vasoactivo o sustancia $P$ (29).

El feocromocitoma puede diagnosticarse con una prueba altamente sensible, como la medición de metanefrinas fraccionadas en orina de 24 horas; también con metanefrinas libres en plasma (4) y niveles totales de catecolaminas urinarias (norepinefrina, epinefrina y dopamina en orina de 24 horas) (4).

\section{Cáncer medular de tiroides}

El cáncer medular de tiroides es un tumor maligno raro de las células C parafoliculares (29). La mayoría de los pacientes son asintomáticos inicialmente. En los pacientes sintomáticos, la diarrea secretora se considera la principal característica clínica; mientras que el rubor en la región facial, en las extremidades superiores y las telangiectasias son menos frecuentes (29). Puede asociarse hasta en un 25\% de los casos con otros tumores endocrinos (feocromocitomas y adenomas paratiroideos), lo que conforma una neoplasia endocrina múltiple.

Ante la sospecha de una masa tiroidea, se debe realizar ultrasonido, como examen de primera línea, ya que aporta información confiable con un buen valor predictivo positivo con respecto a características específicas de los nódulos como posición, tamaño, forma, contenido, márgenes, ecogenicidad, patrón vascular y microcalficaciones (30). Ante la presencia de un nódulo sospechoso, se realizará la biopsia con punción por aspiración con aguja fina para definir el diagnóstico de malignidad (30). La tomografía axial computarizada y la resonancia magnética de cuello y tórax no están indicadas para la evaluación rutinaria de los nódulos tiroideos; pero pueden ser útiles en caso de que el paciente presente síntomas de compresión, a fin de evaluar la afectación de la vía aérea, y en caso de sospecha de extensión retroesternal $(7,31,32)$.

Aunque la calcitonina es un marcador tumoral usado para pacientes con cáncer medular de tiroides, las directrices de la Asociación Americana de Tiroides, revisadas en 2015, señalan que se debe haber juicio clínico en la interpretación de los resultados de la prueba. Las concentraciones séricas pueden ser falsamente altas o bajas en una variedad de enfermedades clínicas, pueden elevarse en niños menores de 3 años y pueden ser más altos en hombres que en mujeres (33). La Asociación Americana de Endocrinólogos Clínicos no hace ninguna recomendación con respecto a la medición rutinaria de la calcitonina sérica en pacientes con nódulos tiroideos, debido a evidencia insuficiente (30).

\section{Tumores endocrinos pancreáticos}

Los tumores neuroendocrinos pancreáticos secretan varias hormonas y péptidos, incluyendo el péptido intestinal vasoactivo, polipéptido inhibidor gástrico, prostaglandina, insulina, gastrina, glucagón, hormona adrenocorticotropa, 
José Manuel Vivas Prieto, Sandra Virginia Caballero Vera, Mateo Echeverry Ramírez.

entre otros (29). La mayoría de los pacientes presenta síntomas relacionados con la hormona que se produce principalmente (29). Es posible que el rubor facial se presente en todas las formas de los tumores neuroendocrinos pancreáticos y se asemeja al del síndrome carcinoide gástrico (29). En el rubor facial crónico, puede haber cambios en la contextura de la piel con presencia de telangiectasias venosas y coloración azulada en la barbilla, la nariz y el área malar de la cara (29). En los vipomas, clásicamente, se presenta el síndrome de Verner-Morrison, que se manifiesta con diarrea acuosa, hipocalemia, aclorhidria y raramente rubor facial $(20,29)$.

Se sospecha el diagnóstico de vipoma en pacientes con diarrea secretora de alto volumen (mayor a $700 \mathrm{ml} /$ día). El diagnóstico se establece mediante una concentración sérica del polipéptido intestinal vasoactivo mayor a $75 \mathrm{pg} / \mathrm{ml}$; sin embargo, se debe confirmar con pruebas repetidas $(4,34)$. Las modalidades imagenológicas incluyen ultrasonografía endoscópica, tomografía computarizada y resonancia magnética (20).

\section{Carcinoma de células renales}

El carcinoma de células renales tiene la tasa de mortalidad más alta en los cánceres genitourinarios (20). Puede causar rubor facial por la secreción de las prostaglandinas o por la vía hipotálamo-pituitaria-adrenal a partir de la liberación de gonadotropinas. Solo el $10 \%$ de los individuos presentan la tríada clásica de hematuria, dolor y masa abdominal palpable en el flanco $(20,35)$. También pueden presentar sintomatología sugestiva de síndrome constitucional, como pérdida de peso, caquexia, pirexia y, en algunos casos, neuromiopatía $(20,36)$.

Para el estudio del carcinoma de célula renal, se realiza inicialmente una ecografía de vías urinarias; si se identifica una lesión de características sólidas, se solicita una tomografía computarizada de alta resolución con medio de contraste (35). El patrón de referencia (gold standard) del diagnóstico es la tomografía computarizada helicoidal, la cual evalúa tanto el tumor primario y su extensión extrarrenal como proporciona información sobre la afectación venosa, crecimiento de adenopatías locorregionales y morfología del riñón contralateral (35). Adicionalmente, se debe realizar hemograma, velocidad de sedimentación globular, fosfatasa alcalina y calcio sérico $(35,37)$.

\section{Mastocitosis}

La mastocitosis es una proliferación neoplásica clonal de los mastocitos que puede afectar muchos órganos (38). Se considera una enfermedad sistémica cuya presentación clínica puede incluir episodios de rubor facial secundarios a la activación mastocitaria, acompañados de taquicardia, hipotensión, síntomas digestivos, etc. Es más frecuente en la edad pediátrica (dos tercios de los casos) que en la adulta (39). La Organización Mundial de la Salud establece los criterios diagnósticos indicados en la tabla $6(40,41)$.

\section{Tabla 6}

\section{Criterios diagnósticos de la OMS para mastocitosis}

\begin{tabular}{|l|l|}
\hline $\begin{array}{l}\text { Criterios } \\
\text { mayores }\end{array}$ & $\begin{array}{l}\text { Presencia de agregados mastocitarios que contengan 15 o más mastocitos } \\
\text { en la biopsia de médula ósea o en otros tejidos. }\end{array}$ \\
\hline $\begin{array}{l}\text { 1. Más del } 25 \% \text { de mastocitos con morfología anormal en la extensión de } \\
\text { médula ósea. } \\
\text { 2. Expresión de los antígenos CD 25, CD 2, o ambos, por citometría de } \\
\text { flujo. } \\
\text { menorites }\end{array}$ & $\begin{array}{l}\text { 3. Triptasa sérica: mayor de } 20 \mathrm{mg} / \mathrm{ml} \text { (no es válido si existe una } \\
\text { hemopatía mieloide asociada). } \\
\text { 4. Presencia de mutación activante del c-kit en los mastocitos de médula } \\
\text { ósea u otro tejido }\end{array}$ \\
\hline $\begin{array}{l}\text { Una mastocitosis se considera sistémica si se cumple un criterio mayor y uno menor, o } 3 \\
\text { criterios menores }\end{array}$ \\
\hline
\end{tabular}

Fuente: adaptado de Sadeghian A, Rouhana $\mathrm{H}$, Oswald-Stumpf B, Boh E. Continuing medical education: Etiologies and management of cutaneous flushing. Malignant causes. J Am Acad Dermatol. 2017;77:405-14.

La Organización Mundial de la Salud reconoce diferentes subtipos de mastocitosis, entre ellos la mastocitosis cutánea (29): usualmente se manifiesta en la edad infantil, y en el $90 \%$ de los casos aparece como urticaria pigmentosa. Los padres consultan por lesiones que varían en tamaño desde unos pocos milímetros hasta algunos centímetros, caracterizadas por una hiperpigmentación pardusca hiperémica (29). 
Las lesiones son fijas, no aparecen y desaparecen en diferentes lugares como la urticaria clásica. Generalmente, no son pruriginosas, pero el prurito puede ser activado por fricción, cambios de temperatura, fiebre, estrés emocional o físico, ejercicio, alcohol y alimentos picantes (40). En el examen físico es importante buscar el llamado signo de Darier (aparición de eritema y lesiones habonosas al frotar las lesiones) (40). Otra presentación menos frecuente asociada a mastocitosis sistémica es la teleangiectasia macularis eruptiva perstans, en la que la piel tiene máculas telangiectásicas planas en distribución generalizada (42).

Las manifestaciones clínicas cutáneas, cuando se presentan en la edad adulta, tienden a agregarse en áreas propensas a la irritación, como la parte superior de muslos y axilas, y por lo general no aparecen en áreas expuestas al sol como la cara y las manos (40).

El diagnóstico inicialmente es clínico, si se presentan las lesiones características de urticaria pigmentosa, que pueden estar asociadas a episodios de rubor facial o anafilaxis (27). Se debe realizar una biopsia de las lesiones en la piel para confirmar el diagnóstico (27). La presencia de manifestaciones cutáneas no descarta la afectación sistémica. Se deben realizar estudios de extensión para descartar el diagnóstico de mastocitosis sistémica (27).

La triptasa es un marcador de la degranulación de los mastocitos liberados en paralelo con la histamina. Se eleva típicamente en pacientes con mastocitosis sistémica; mientras que los pacientes con mastocitosis cutánea, por lo general, presentan concentraciones normales de triptasa sérica (27). La medición de nmetilhistamina en la orina de 24 horas y metabolitos D2 de prostaglandina son útiles para el diagnóstico (4). Sin embargo, estos marcadores séricos no son de fácil acceso en el ámbito de la atención primaria, lo que limita su utilidad en la práctica clínica diaria.
Rubor facial transitorio relacionado con el manejo de cáncer de próstata

El cáncer de próstata es el principal cáncer en el hombre. Cerca del $70 \%$ de los casos son diagnosticados en hombres mayores de 65 años de edad (43). Dado que la testosterona es esencial para el crecimiento de la próstata, la terapia de deprivación de andrógenos, ya sea con la orquiectomía bilateral o la administración del análogo de la hormona liberadora de gonadotropina, es la terapia inicial recomendada para el cáncer metastásico o localmente avanzado (43). La terapia hormonal genera efectos adversos como sofocos, pérdida de la libido o disfunción eréctil y fatiga, lo cual afecta la calidad de vida de los pacientes (43). Los sofocos se presentan en el 34\%-80\% de los hombres (43); el mecanismo no se conoce muy bien, pero se cree que los cambios repentinos de las cantidades de los esteroides sexuales alteran la función de los neurotransmisores cerebrales, como serotonina, noradrenalina y betaendorfinas, generando inestabilidad en el centro de la termorregulación del hipotálamo. Algunos estudios han mostrado que aproximadamente el $40 \%$ de los pacientes persiste con rubor facial posterior a 8 años del tratamiento $(44,45)$.

\section{Manejo del rubor facial resistente}

En el manejo del rubor facial resistente a las terapias de primera línea se han descrito las siguientes medidas coadyuvantes:

Tratamiento psicológico: Se sugiere la terapia cognitivo-conductual en fobia social (46).

Simpatectomía superior: indicada en aquellos pacientes para quienes el tratamiento médico ha fracasado, y para quienes la percepción del rubor facial les genere un efecto negativo en su calidad de vida (8). En una revisión sistemática (47), donde se incluyeron 1369 pacientes, se mostró que el 84,02\% de los pacientes tenía una satisfacción completa a la simpatectomía, con una tasa de éxito en el 78,30\% de los pacientes. Los estudios mostraron efectos secundarios como la sudoración compensatoria en el $74,18 \%$, 
(IC 95\%: 58,10\%-90,26\%), sudoración gustativa 24,42\% (IC 95\%: 12,22\%-36,61\%), y menos del $2 \%$ presentaron síndrome de Horner $(46,47)$. Ocasionalmente, se puede presentar el fenómeno de Arlequín, caracterizado por denervación asimétrica, con enrojecimiento hemifacial y palidez contralateral (46).

Toxina botulínica: puede ser un tratamiento eficaz y seguro para el eritema refractario y rosácea, cuando el tratamiento de primera línea ha fracasado, aunque faltan estudios que incluyan una muestra representativa y evidencia clínica que muestre su efectividad (48).

\section{Conclusión}

El rubor facial transitorio representa un reto para el médico de atención primaria, quien debe tener en cuenta los diagnósticos diferenciales tanto de etiología benigna como maligna, para lo cual deben buscarse de forma activa señales de alarma en la anamnesis y en el examen físico, lo cual le permitirá, desde la atención primaria, hacer un abordaje completo sobre el origen de la sintomatología y así poder definir un enfoque terapéutico o según el caso remitir al paciente de manera oportuna a la especialidad pertinente.

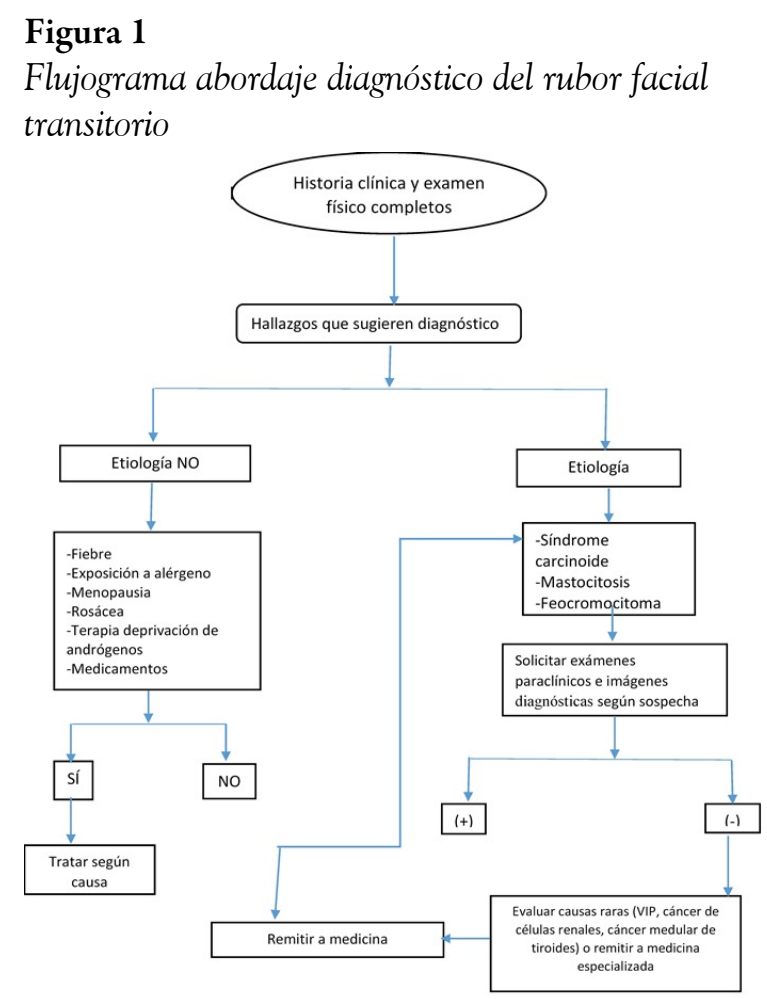

\section{Agradecimientos}

A la doctora Luz Helena Alba Talero, profesora asociada Facultad de Medicina, Pontificia Universidad Javeriana, Departamento de Medicina Preventiva y Social, y a la doctora María Fernanda Ordoñez Rubiano, jefe de residentes, especialidad en Dermatología, Facultad de Medicina, Universidad Militar Nueva Granada, por su valiosa revisión y las sugerencias que hicieron acerca de este trabajo, lo cual ayudó a mejorar su calidad.

\section{Referencias}

1. Batalla Cebey A, De la Torre Fraga C. Rubor facial transitorio: Metodología diagnóstica y tratamiento. Piel. 2012;27(7):390-400.

2. Kaupp-Roberts S, Srirajaskanthan R, Ramage JK. Symptoms and quality of life in gastroenteropancreatic 
neuroendocrine tumors. Cit EMJ Oncol. 2015;3(1):34-40.

3. Salleras RM, Lamas Doménech N. Tratamiento del rubor facial (flushing). Piel. 2014;29(9):587-91.

4. Izikson L, English JC, Zirwas MJ. The flushing patient: Differential diagnosis, workup, and treatment. J Am Acad Dermatol. 2006;55(2):193-208.

5. Nasr C. Flushing. En: Current clinical medicine. 2nd ed. Cleveland: Elsevier; 2010. p. 369-75.

6. Luft FC. Asian flushing presents opportunities for disease prevention. J Mol Med. 2016;94(11):1195-7. doi: http://dx.doi.org/10.1007/s00109. 016-1470-8

7. Yale SH, Vasudeva S, Mazza JJ, Rolak L, Arrowood J, Stichert S, et al. Disorders of flushing. Compr Ther. 2005;31(1):59-71.

8. Scholing A, Emmelkamp PM. Cognitive and behavioural treatments of fear of blushing, sweating or trembling. Behav Res Ther. 1993;31:155-70.

9. Nicolaou M, Paes T, Wakelin S. Blushing: An embarrassing condition, but treatable. Lancet. 2006;367 (9519):1297-9.

10. Drott C. Results of endoscopic thoracic sympathectomy (ETS) on hyperhidrosis, facial blushing, angina pectoris, vascular disorders and pain syndromes of the hand and arm. Clin Auton Res. 2003;13:1-1.

11. Tüzün Y, Wolf R, Kutlubay Z, Karakuş Ö, Engin B. Rosacea and rhinophyma. Clin Dermatol. 2014;32(1):35-46.

12. Molina AL, Londoño Á, Escobar SM, Benítez M. Guías clínicas para el tratamiento de la rosácea. Rev Asoc Colomb Dermatol. 2012 oct;20(4):339-64.
13. Sadeghian A, Rouhana $\mathrm{H}$, Oswald-Stumpf B, Boh E. Continuing medical education: Etiologies and management of cutaneous flushing. Nonmalignant causes. J Am Acad Dermatol 2017;77:391-402.

14. Torrens MB. Rosácea y rinofima. AMF. 2013;9(7):397-401.

15. Schaller M, Schöfer H, Homey B, Hofmann M., Gieler U, Lehmann P, et al. Rosacea management: Update on general measures and topical treatment options. J Dtsch Dermatol Ges. 2016;14:17-27.

16. Troielli P, González FM, Ríos JM, Vásquez OT., Pabón JG, Ibarra M, et al. Actualización y recomendaciones para el diagnóstico y tratamiento de la rosácea en Latinoamérica. Med Cutan Iber Lat Am. 2016;44(supl 1):S7-26.

17. Takahashi TA, Johnson KM. Menopause. Med Clin North Am. 2015;99(3):521-34.

18. Freeman EW. Hot flushes and the menopause: How long should they be expected to last? Maturitas. 2014;78(3):153-4.

19. Ikizoglu G. Red face revisited: Flushing. Clin Dermatol. 2014;32(6):800-8.

20. Goodman NF, Cobin RH, Ginzburg SB, Katz IA, Woode DE. American Association of Clinical Endocrinologists Medical Guidelines for Clinical Practice for the Diagnosis and Treatment of Menopause. Endocrine Practice. 2011;17:1-25.

21. Hauk L. Acog releases clinical guidelines on management of menopausal symptoms. Am Fam Physician. 2014;90(5):338-40.

22. Sassarini J, Lumsden MA. Vascular function and cardiovascular risk factors in women with severe flushing. Maturitas. 2015;80(4):379-83. 
23. Tuomikoski P, Savolainen-Peltonen H. Vasomotor symptoms and metabolic syndrome. Maturitas. 2017;97:61-5.

24. Hernandez L, Papalia S, Pujalte GA. Anaphylaxis. Prim Care Clin off Pract. 2016;43(3):477-85.

25. Adams KE, Rans TS. Adverse reactions to alcohol and alcoholic beverages. Ann Allergy Asthma Immunol. 2013;111(6):439-45.

26. Callejas MA. Blushing: pasado, presente y futuro. Arch Bronconeumol. 2009;45:65-7.

27. Huguet I, Grossman A. Flushing: current concepts. Eur J Endocrinol. 2017;177(5):R219-29.

28. Jayasena CN, Dhillo WS. Carcinoid syndrome and neuroendocrine tumours. Medicine. 2013;41;10:566-9.

29. Hannah SF, Stratakis Ca, Koch Ca. Flushing in (neuro) endocrinology. Rev Endocr Metab Disord. 2016;17(3):373-80.

30. Franco UC, Pardo BF, Laborda HR, Pérez LC. Usefulness of ultrasonography is the evaluation of thyroid nodules. Radiología. 2016;58(5):380-8.

31. Herber GM, Mishra GD. Early severe vasomotor menopausal symptoms are associated with diabetes. Menopause. 2014;21(8):855-60.

32. Wells SA, Asa SL, Dralle $\mathrm{H}$, et al. Revised American Thyroid Association Guidelines for the Management of Medullary Thyroid Carcinoma: The American Thyroid Association Guidelines Task Force on Medullary Thyroid Carcinoma. Thyroid. 2015;25(6):567-610. doi: htt ps://doi.org/10.1089/thy.2014.0335.

33. Paschou SA, Vryonidou A, Goulis DG. Thyroid nodules: A guide to assessment, treatment and follow-up. Maturitas. 2017;96:1-9.
34. Fazio SB. Approach to flushing in adults [internet]. 2016. Disponible en: http://www.uptodate.com/contents /approach-to-flushing-in-adults.

35. Quiroga Matamoros W, Fernández F, Citarella Otero D, Rangel J, Estrada Guerrero A, Patiño ID. Guía de manejo del carcinoma de células renales. Urol Colomb. 2016;25:169-89.

36. Ljungberg B, Damian HC, Marcus KA, Axel MS, Peter Mulders FA, Patard J, et al. Guía del carcinoma de células renales. Actas Urol Esp. 2009;33(3):270-79.

37. Hutson TE. Targeted therapies for the treatment of metastatic renal cell carcinoma: Clinical evidence. Oncologist. 2011;16(Suppl 2):14-22.

38. Sadeghian A, Rouhana $H$, Oswald-Stumpf B, Boh E. Continuing medical education: Etiologies and management of cutaneous flushing. Malignant causes. J Am Acad Dermatol 2017;77:405-14.

39. Akin C, Valent P. Diagnostic criteria and classification of mastocytosis in 2014. Immunol Allergy Clin North Am. 2014;34(2):207-18.

40. Wolin EM. The expanding role of somatostatin analogs in the management of neuroendocrine tumors. Gastrointest Cancer Res. 2012;5(5):161-8.

41. Jones MR, Vertovse KS, Jorgensen JL, Manshoveri T, Luther R, Jones $\mathrm{DM}$, et al. Utility of the World Health Organization classification criteria for the diagnosis of systemic mastocytosis in bone marrow. Mod Pathol. 2009;22(1):50-7.

42. Izikson L, English JC, Zirwas MJ. The flushing patient: Differential diagnosis, workup, and treatment. J Am Acad Dermatol. 2006;55(2):193-208. 
43. Frisk J. Review: Managing hot flushes in men after prostate cancer -A systematic review. Maturitas. 2010;65:15-22.

44. Ahmadi $\mathrm{H}$, Daneshmand S. Androgen deprivation therapy: Evidence-based management of side effects. BJU Int. 2013;111(4):543-8. doi: https://doi.org/10.1111/j.1464-410 X.2012.11774.x.

45. Frisk JW, Hammar ML, Ingvar $\mathrm{M}$, et al. How long do the effects of acupuncture on hot flashes persist in cancer patients? Suppoert Care Cancer. 2014;22(5):1409-15.

46. Girish G, D'souza RE, D'souza P, Lewis MG, Baker DM. Role of surgical thoracic sympathetic interruption in treatment of facial blushing: a systematic review. Postgrad Med. 2017;129(2):267-75. doi: https://doi.or g/10.1080/00325481.2017.1283207.

47. Kristian S, Christer D. Facial blushing: Patient selection and longterm results. Thorac Surg Clin. 2016;26(4):459-63. doi: https://doi.org /10.1016/j.thorsurg.2016.06.011

48. Park KY, Hyun MY, Jeong SY, Kim BJ, Kim MN., Hong CK. Botulinum toxin for the treatment of refractory erythema and flushing of rosacea. Dermatology. 2015;230(4):299-301. 